\title{
Intraoperative Evaluation of Sentinel Lymph Nodes for Metastatic Breast Carcinoma by Imprint Cytology
}

Andrew J. Creager, M.D., Kim R. Geisinger, M.D., Stephen A. Shiver, M.D., Nancy D. Perrier, M.D., Perry Shen, M.D., Jo Ann Shaw, M.D., Peter R. Young, M.D., Edward A. Levine, M.D.

Department of Pathology (AJC), Duke University Medical Center, Durham, NC; Department of Pathology (KRG) and Surgical Oncology Service and Department of Surgery (SAS, NDP, PS, EAL), Wake Forest University School of Medicine, Winston-Salem, NC; Greensboro Pathology Associates, PA (JS), and Central Carolina Surgery, PA (PRY), Moses Cone Health System, Greensboro, NC

Background: The increasing utilization of lymphatic mapping techniques for breast carcinoma has made intraoperative evaluation of sentinel lymph nodes attractive. Axillary lymph node dissection can be performed during the initial surgery if the sentinel lymph node is positive, potentially avoiding a second operative procedure. At present the optimal technique for rapid sentinel lymph node assessment has not been determined. Both frozen sectioning and intraoperative imprint cytology are used for rapid intraoperative sentinel lymph node evaluation at many institutions. The purpose of this study is to evaluate experience with imprint cytology for intraoperative evaluation of sentinel lymph nodes in patients with breast cancer. Methods: A retrospective review of the intraoperative imprint cytology results of 678 sentinel lymph node mappings for breast carcinoma was performed. Sentinel nodes were evaluated intraoperatively by either bisecting or slicing the sentinel node into $4 \mathrm{~mm}$ sections. Imprints were made of each cut surface and stained with H\&E and/or Diff-Quik. Permanent sections were evaluated with up to four H\&E stained levels and cytokeratin immunohistochemistry. Intraoperative imprint cytology results were compared with final histologic results. Results: The sensitivity of imprint cytology was $53 \%$, specificity was $98 \%$, positive predictive value was $94 \%$, negative predictive value was $82 \%$ and accuracy was $84 \%$. The sensitivity for detecting macrometastases ( more than $2 \mathrm{~mm}$ ) was significantly better than for detecting micrometastases ( $\leq 2 \mathrm{~mm}$ ), 81 versus $21 \%$, respectively $(P<00001)$. Conclusions: The sensitivity and

Copyright () 2002 by The United States and Canadian Academy of Pathology, Inc.

VOL. 15, NO. 11, P. 1140, 2002 Printed in the U.S.A.

Date of acceptance: August 21, 2002.

Address reprint requests to: Andrew J. Creager, M.D., Department of Pathology, Duke University Medical Center, DUMC 3712, Durham, NC 27710; e-mail: Creag001@mc.duke.edu; fax: 919-684-8689.

DOI: 10.1097/01.MP.0000036385.54165.E1 specificity of imprint cytology are similar to that of intraoperative frozen section evaluation. Imprint cytology is therefore a viable alternative to frozen sectioning when intraoperative evaluation is required. If sentinel lymph node micrometastasis is used to determine the need for further lymphadenectomy, more sensitive intraoperative methods will be needed to avoid a second operation.

KEY WORDS: Breast carcinoma, Cytopathology, Imprint Metastatic, Sentinel lymph node.

Mod Pathol 2002;15(11):1140-1147

The single most important predictor of outcome for women with breast carcinoma is the status of the regional lymph nodes (1-5). Traditionally, axillary lymph node status has been evaluated by routine axillary lymph node dissection accompanying lumpectomy or mastectomy specimens. Axillary lymph node dissection is used to obtain precise staging data, provide local control for patients with metastatic breast carcinoma and for selection of adjuvant therapy (5-11). Unfortunately, the only patients who are likely to derive therapeutic benefit from axillary lymph node dissection are those with positive nodes, approximately $40 \%$ of those undergoing axillary dissection (12-16). Axillary lymph node dissection is associated with considerable chronic morbidity including lymphedema, neurologic damage to the brachial plexus, joint stiffness and, rarely, angiosarcoma (17-20). Sentinel lymph node biopsy is a highly accurate predictor of the overall axillary status and has both a high sensitivity and specificity, especially when primary tumors are small (21). Sentinel lymph node mapping is attractive as it may identify a population of breast cancer patients that may benefit from axillary lymph node dissection. More importantly, it may identify those patients in whom axillary dissection and its associated morbidity may be avoided while still providing accurate staging data (21). 
One of the most exciting current roles of sentinel lymph node biopsy is the ability to stage patients intraoperatively, thus providing a single operative procedure for sentinel lymph node biopsy and axillary dissection, should the sentinel node be found to contain metastatic carcinoma. Currently intraoperative evaluation is performed using intraoperative imprint cytology (22-29) and intraoperative frozen sectioning $(13,30-38)$ and in some institutions the use of rapid intraoperative cytokeratin staining is used in conjunction with these methods $(31,39$, 40). In addition, combined imprint cytology and frozen sectioning are used at some institutions (12, 41,42 ). In the present study we analyze the utility of imprint cytology in the intraoperative evaluation of sentinel nodes for metastatic breast carcinoma in 678 consecutive lymph node mappings performed at both an academic medical center and a community hospital.

\section{METHODS}

\section{Patients and Data Collection}

All patients undergoing SLN mapping for cancer of the breast from May 1997 to May 2001 from the Moses H. Cone Hospital, Greensboro, NC and Wake Forest University Baptist Medical Center, WinstonSalem, NC were reviewed. A chart review of each patient was performed for the following data: age, sex, method of tumor detection, type of breast and axillary surgical procedure. Primary tumor data compiled included: size, type, and histologic grade. Lymph node data gathered included, number of sentinel nodes and non-sentinel nodes obtained, number of each containing metastatic tumor and size of largest metastatic deposits. Tumor size was recorded according to the guidelines of the American Joint Committee on Cancer (43). Histopathologic grade was based on the modified BloomRichardson system (46).

\section{Surgical Protocol}

A standard protocol was used to identify the sentinel nodes. The patient's tumor bed was injected with filtered technetium sulfur colloid (0.5-1.0 mCi) preoperatively on the day of surgery. Intraparenchymal peritumoral injections were performed using palpation as a guide. Injections were guided by a localization wire for otherwise non-palpable lesions. Patients who had previously undergone an excisional biopsy received injections adjacent to the biopsy cavity. A lymphoscintigram was obtained in selected patients early in the experience before arrival in the operative suite. A gamma probe (Neoprobe 2000) was used intraoperatively to detect the sentinel node. In addition, in all cases peri- lesional injections of isosulfan blue were used intraoperatively to provide visual identification of the sentinel node. After injection of the blue dye the breast was massaged for 5 minutes before incision. The sentinel nodes were then harvested and sent fresh to pathology for intraoperative and permanent section evaluation. Complete axillary lymph node dissection was performed only if the SLN contained tumor cells, unless the patient was on a research protocol mandating completion lymphadenectomy.

\section{Pathologic Examination}

Excised lymph nodes submitted for intraoperative evaluation were examined using one of two methods depending on which institution was performing the evaluation. In the first method, the sentinel node was bisected along the long axis. Care was taken to obtain complete cross sections of the maximum diameter, preferably including the hilum and the marginal sinus. For each lymph node half, a pair of imprints was made by gently touching the cut surface of the sentinel node to a glass slide. One imprint from each pair was air dried and stained with the Diff-Quik stain. The second imprint from each surface was immediately fixed in $10 \%$ buffered formalin and then stained with $\mathrm{H} \& \mathrm{E}$ in the usual manner. In the second method, the SLN was sliced into $4 \mathrm{~mm}$ slices and imprints were made of each cut surface, air-dried and stained with Diff-Quik.

Imprints were then reviewed intraoperatively by either a board certified cytopathologist or a pathologist with informal cytologic training. Diagnostic categories included positive or negative for tumor or atypical cells present. In the early part of the study a diagnosis of both positive and atypical cells resulted in completion axillary lymph node dissection; however, later in the study to minimize the possibility of a false positive diagnosis the atypical diagnostic category was considered negative at the time of surgery by the surgeon and completion dissections were delayed until the results of permanent section evaluation became available. After an interpretation was rendered and the surgical team was notified, the sentinel node was fixed in $10 \%$ formalin, processed in the usual manner and paraffin embedded. Again, depending on the institution, two methodologies were employed for permanent section evaluation of the sentinel node. At the first institution a single $\mathrm{H} \& \mathrm{E}$ stained section from the sentinel node was cut from the paraffin block and examined. If initial review of the H\&E stained section was negative, a sentinel node protocol consisting of an additional three H\&E stained levels cut at $50 \mu$ intervals in conjunction with immunohistochemical stains for cytokeratin (AE1/AE3, Dako, Carpinteria, CA) was performed on the first of the three levels (unless the patient was on a research 
protocol that proscribed such analysis). At the second institution sentinel nodes were first evaluated by means of four H\&E stained levels cut at $50 \mu$ intervals. If these were negative, immunohistochemical stains for cytokeratin (AE1/AE3) were evaluated on a final level. For all cases, immunohistochemical studies were carried out using the avidin-biotin-peroxidase complex (ABC) method described previously (45). Immunohistochemical stains for cytokeratin were considered positive if strong immunoreactivity in cell clusters or individual cells that demonstrated anatomic and cytologic features of metastatic tumor cells were identified.

When discrepancies existed between the intraoperative and permanent section results, the slides were reviewed by an independent pathologist in an attempt to determine the cause of the discrepancy. There was no standard protocol for the permanent section evaluation of false positive sentinel node imprints and this was left to the discretion of the pathologist. However, additional H\&E stained levels were obtained for all false positives, including sectioning the block completely in some instances. Additionally, for most false positives additional levels were evaluated with cytokeratin immunohistochemistry.

When non-sentinel nodes were obtained, they were examined using standard pathologic techniques. If greater than $4 \mathrm{~mm}$ in width, non-sentinel nodes were sectioned, if less than $4 \mathrm{~mm}$ nonsentinel nodes were submitted whole. Routinely, a single H\&E stained section of the non-sentinel nodes was examined and in several cases multiple levels were obtained in an attempt to verify the presence of metastases.

\section{Statistics}

Chi-squared and Fisher exact tests (when cell counts were small) were used to assess differences in clinical and pathologic factors between those with and those without sentinel node performed, and to assess the association between pathologic factors and intraoperative imprint cytology results. Sensitivity was defined as the percentage of positive imprint cytology results among those with positive permanent pathology. Specificity was defined as the percentage of negative imprint cytology results among those with negative permanent pathology. Two false positive categories existed. The first definition included cases called positive intraoperatively in which permanent section evaluation failed to detect sentinel node metastases. The second false positive category included cases called atypical cells intraoperatively, triggering a completion lymph node dissection, when the permanent section evaluation was negative. The false-negative rate was defined as the number of false-negative intraoperative imprint results divided by the sum of the false-negative and true-positive results. For the purposes of this manuscript, statistical significance was defined as a $P$ value of less than 0.05 .

\section{RESULTS}

\section{Patient and Primary Tumor Data}

We attempted sentinel lymph node mapping procedures in 678 consecutive patients and a sentinel node was identified in 646 (95.3\%). These 646 consecutive cases with intraoperative imprint cytologic diagnosis of the sentinel node were all evaluated as per protocol. The patients ranged in age from 21 to 88 years (mean 58 years). Only two men were present in the series. Breast-conserving surgery was performed in $73 \%$ of patients and the remaining $27 \%$ underwent mastectomy.

Primary invasive tumors ranged from 0.1 to 8.8 $\mathrm{cm}$ (mean $1.7 \mathrm{~cm}$ ). Tumor representation was as follows: 513 usual invasive ductal carcinomas, 25 special types of invasive ductal carcinoma (15 mucinous, 8 tubular, 1 cribiform, and 1 papillary), 17 cases of pure ductal carcinoma in situ, 61 cases of invasive lobular carcinoma, which included 54 classic type (grade 1) and 7 pleomorphic type (grade 2-3), a single case of lobular carcinoma in situ, 27 carcinomas with mixed ductal and lobular features, and 2 metaplastic carcinomas (Table 1). Histologic grades of invasive carcinomas included, 158 grade 1,325 grade 2 and 145 grade 3 tumors. For the 18 in situ carcinomas only nuclear grade was reported.

\section{Sentinel Lymph Nodes}

From 646 patients a total of 1,255 sentinel nodes were evaluated by intraoperative imprint cytology (median, 2.0 SLN/patient). A separate intraoperative diagnosis was generated for each lymph node. Results are reported on a per patient basis.

TABLE 1. Represented Tumors by Histologic Type

\begin{tabular}{lc}
\hline \multicolumn{1}{c}{ Type of Carcinoma } & Number of Cases \\
\hline Ductal carcinoma & 17 \\
Pure in situ carcinoma & 513 \\
Invasive, NOS* & 15 \\
Mucinous & 8 \\
Tubular & 1 \\
Papillary & 1 \\
Cribiform & 1 \\
Lobular carcinoma & 54 \\
Pure in situ carcinoma & 7 \\
Classic & \\
Pleomorphic & 27 \\
Other types & 2 \\
Mixed ductal and lobular features & \\
Metaplastic carcinoma & 646 \\
Total &
\end{tabular}

* NOS, not otherwise specified. 
TABLE 2. Comparison of Intraoperative Imprint Cytologic Evaluation Relative to Permanent Section Evaluation by Tumor Size Classification

\begin{tabular}{|c|c|c|c|c|c|c|c|}
\hline Size & $\begin{array}{l}\text { Number } \\
\text { of } \\
\text { Patients }\end{array}$ & $\begin{array}{c}\text { Number of Patients } \\
\text { with }+ \text { SLN on } \\
\text { Permanents }\end{array}$ & $\begin{array}{c}\text { Number of Patients with } \\
+ \text { SLN by IIC }\end{array}$ & $\begin{array}{l}\text { Number False }+ \\
\text { by IIC }\end{array}$ & Accuracy (\%) & Sensitivity (\%) & Specificity (\%) \\
\hline Tis & 18 & 2 & 0 & 1 & 83 & 0 & 94 \\
\hline T1mic & 21 & 2 & 1 & 1 & 86 & 50 & 95 \\
\hline T1a & 42 & 6 & 6 & 0 & 100 & 100 & 100 \\
\hline T1b & 143 & 30 & 12 & 0 & 87 & 40 & 100 \\
\hline T1c & 255 & 86 & 47 & 4 & 83 & 55 & 98 \\
\hline $\mathrm{T} 2$ & 159 & 73 & 39 & 1 & 89 & 53 & 99 \\
\hline T3 & 8 & 5 & 4 & 0 & 88 & 80 & 100 \\
\hline Total & 646 & 204 & 109 & 7 & 84 & 53 & 98 \\
\hline
\end{tabular}

SLN, sentinel lymph node, IIC, intraoperative imprint cytology.

The accuracy of intraoperative imprint cytology compared with permanent section evaluation of the sentinel node was $84 \%$ (Table 2). Of 204 patients with positive sentinel nodes by permanent section evaluation, there were 95 false negative intraoperative imprint evaluations (Sensitivity, 53\%). Sensitivity of detecting metastatic disease by imprint cytology was significantly higher for macrometastatic disease (metastases more than $0.2 \mathrm{~cm}$ ) than micrometastatic disease (metastases $\leq 0.2 \mathrm{~cm})(P<$ .00001) (Table 3). There was a statistically significant association between accuracy of intraoperative imprint cytology and tumor size $(P=.0123)$ (Table 2). There was no difference in specificity associated with tumor size $(P=.0261)$.

The sensitivities of detecting pure invasive ductal carcinoma, invasive mixed ductal and lobular carcinoma and pure invasive lobular carcinoma were $53 \%, 60 \%$ and $52 \%$, respectively. No statistically significant difference was observed in detection of pure invasive ductal and lobular carcinoma by intraoperative imprint cytology $(P=.9926)$ (Table 4$)$.

TABLE 3. Sensitivity of Intraoperative Imprint Cytology Relative Size of Metastasis

\begin{tabular}{lccc}
\hline \multicolumn{1}{c}{$\begin{array}{c}\text { Size of } \\
\text { Metastasis }\end{array}$} & $\begin{array}{c}\text { Number of Patients } \\
\text { with + SLN on } \\
\text { Permanents }\end{array}$ & $\begin{array}{c}\text { Number of Patients } \\
\text { with + SLN by IIC }\end{array}$ & $\begin{array}{c}\text { Sensitivity } \\
(\%)\end{array}$ \\
\hline $0.2 \mathrm{~cm}$ & 97 & 21 & 21 \\
$>0.2 \mathrm{~cm} 2.0 \mathrm{~cm}$ & 100 & 81 & 81 \\
$>2.0 \mathrm{~cm}$ & 7 & 7 & 100 \\
\hline
\end{tabular}

SLN, sentinel lymph node; IIC, intraoperative imprint cytology.
The sensitivity of detecting a positive sentinel node by imprint cytology was associated with increasing histologic grade. Sensitivities were $33 \%$, $54 \%$ and $65 \%$ for grade 1 through 3 tumors, respectively (Table 5). This finding is likely a result of larger metastases being associated with higher histologic grade tumors. No statistically significant difference was observed between the different sectioning methodologies used to evaluate the sentinel node at the two different institutions (Table 6.)

Seven false positive intraoperative imprint evaluations resulting in axillary dissections were identified (specificity 98\%) (Fig. 1). Four false positive were diagnosed intraoperatively as atypical cells present while the remaining three cases were diagnosed as positive. Primary tumors associated with false positives included five invasive ductal carcinomas (one of which was a mucinous carcinoma), one invasive carcinoma with mixed ductal and lobular features and one case of pure lobular carcinoma in situ adjacent to but not associated with a fibroadenoma. The four cases diagnosed as atypical cells intraoperatively were considered on retrospective review to be negative with the atypical cells likely representing histiocytes, a well-recognized phenomenon (46). The three false positive cases diagnosed as positive intraoperatively demonstrated unequivocal malignant cells on retrospective review of the imprints. Of these three cases, one excision demonstrated a benign fibroadenoma with lobular carcinoma in situ and no invasive carcinoma, another case demonstrated only adipose tis-

TABLE 4. Comparison of Imprint Cytologic Evaluation Relative to Permanent Section Evaluation for Different Histologic Types of Invasive Carcinoma

\begin{tabular}{|c|c|c|c|c|c|c|c|}
\hline $\begin{array}{c}\text { Type of } \\
\text { Invasive } \\
\text { Carcinoma }\end{array}$ & $\begin{array}{c}\text { Number } \\
\text { of } \\
\text { Patients }\end{array}$ & $\begin{array}{l}\text { Number of Patients with } \\
+ \text { SLN on Permanents }\end{array}$ & $\begin{array}{l}\text { Number of Patients } \\
\text { with + SLN by IIC }\end{array}$ & $\begin{array}{c}\text { Number False }+ \\
\text { by IIC }\end{array}$ & Accuracy (\%) & Sensitivity (\%) & Specificity (\%) \\
\hline Lobular & 62 & 23 & 12 & 1 & 80 & 52 & 98 \\
\hline Ductal & 555 & 170 & 90 & 5 & 83 & 53 & 98 \\
\hline $\begin{array}{l}\text { Mixed } \\
\text { ductal/lobular }\end{array}$ & 27 & 10 & 6 & 0 & 85 & 60 & 100 \\
\hline
\end{tabular}

SLN, sentinel lymph node; IIC, intraoperative imprint cytology. 
TABLE 5. Comparison of Intraoperative Imprint Cytologic Evaluation Relative to Permanent Section Evaluation for Different Histologic Grades of Invasive Carcinoma

\begin{tabular}{|c|c|c|c|c|c|c|c|}
\hline $\begin{array}{c}\text { Grade of } \\
\text { Invasive } \\
\text { Carcinoma }\end{array}$ & $\begin{array}{c}\text { Number } \\
\text { of } \\
\text { Patients }\end{array}$ & $\begin{array}{l}\text { Number of Patients with } \\
+ \text { SLN on Permanents }\end{array}$ & $\begin{array}{l}\text { Number of Patients } \\
\text { with + SLN by IIC }\end{array}$ & $\begin{array}{l}\text { Number False }+ \\
\text { by IIC }\end{array}$ & Accuracy (\%) & Sensitivity (\%) & Specificity (\%) \\
\hline 1 & 158 & 40 & 13 & 1 & 83 & 33 & 99 \\
\hline 2 & 321 & 102 & 55 & 2 & 85 & 54 & 99 \\
\hline 3 & 145 & 63 & 41 & 3 & 83 & 64 & 97 \\
\hline
\end{tabular}

SLN, sentinel lymph node; IIC, intraoperative imprint cytology.

TABLE 6. Comparison of Intraoperative Imprint Cytologic Evaluation Relative to Permanent Section Evaluation for Different Methods of Sentinel Lymph Node Evaluation

\begin{tabular}{|c|c|c|c|c|c|c|c|}
\hline $\begin{array}{l}\text { Method of } \\
\text { Evaluation }\end{array}$ & $\begin{array}{l}\text { Number } \\
\text { of } \\
\text { Patients }\end{array}$ & $\begin{array}{l}\text { Number of Patients with } \\
+ \text { SLN on Permanents }\end{array}$ & $\begin{array}{l}\text { Number of Patients } \\
\text { with + SLN by IIC }\end{array}$ & $\begin{array}{l}\text { Number False }+ \\
\text { by IIC }\end{array}$ & Accuracy (\%) & Sensitivity (\%) & Specificity (\%) \\
\hline Bisected & 126 & 34 & 19 & 0 & 88 & 56 & 100 \\
\hline $\begin{array}{l}4 \mathrm{~mm} \\
\text { slices }\end{array}$ & 520 & 170 & 90 & 7 & 83 & 53 & 98 \\
\hline
\end{tabular}

SLN, sentinel lymph node; IIC, intraoperative imprint cytology.

sue submitted as the sentinel node, and the last case did not demonstrate a metastasis on permanent section evaluation although malignant cells, morphologically similar to the patients primary breast tumor, were clearly present on the imprint slides. In the first two cases the origin of the malignant cells is unclear, although one pathologist raised the possibility of bench contamination with tumor cells from another case. In the last case we believe a micrometastasis was sampled intraoperatively and was sectioned through on permanent section evaluation (true positive intraoperative evaluation). No non-sentinel nodes were positive in any of these seven cases.

Two of 25 patients with special types of low-grade invasive ductal carcinoma had a positive sentinel node $(8 \%)$. One patient had a $0.8 \mathrm{~cm}$ invasive papillary carcinoma, one patient had a $0.5 \mathrm{~cm}$ invasive tubular carcinoma. Both of these patients had metastases greater than $0.2 \mathrm{~cm}$. A single false positive intraoperative imprint was reported in a patient with mucinous carcinoma. On retrospective review of this case the atypical cells appeared to represent aggregates of atypical histiocytes with increased nuclear to cytoplasmic ratios.

\section{Non-Sentinel Lymph Nodes}

A total of 2948 non-sentinel lymph nodes were identified in 285 patients (median 10/patient). 199 non-sentinel nodes from 56 patients harbored metastatic carcinoma. Non-sentinel nodes were obtained in 132/204 cases for which sentinel nodes were positive. Forty-four of these (33\%) harbored carcinoma in the non-sentinel node. In seven patients with negative sentinel node, a non-sentinel node harbored carcinoma. The accuracy of the sentinel lymph node mapping procedure was therefore estimated at $98.9 \%(639 / 646)$.

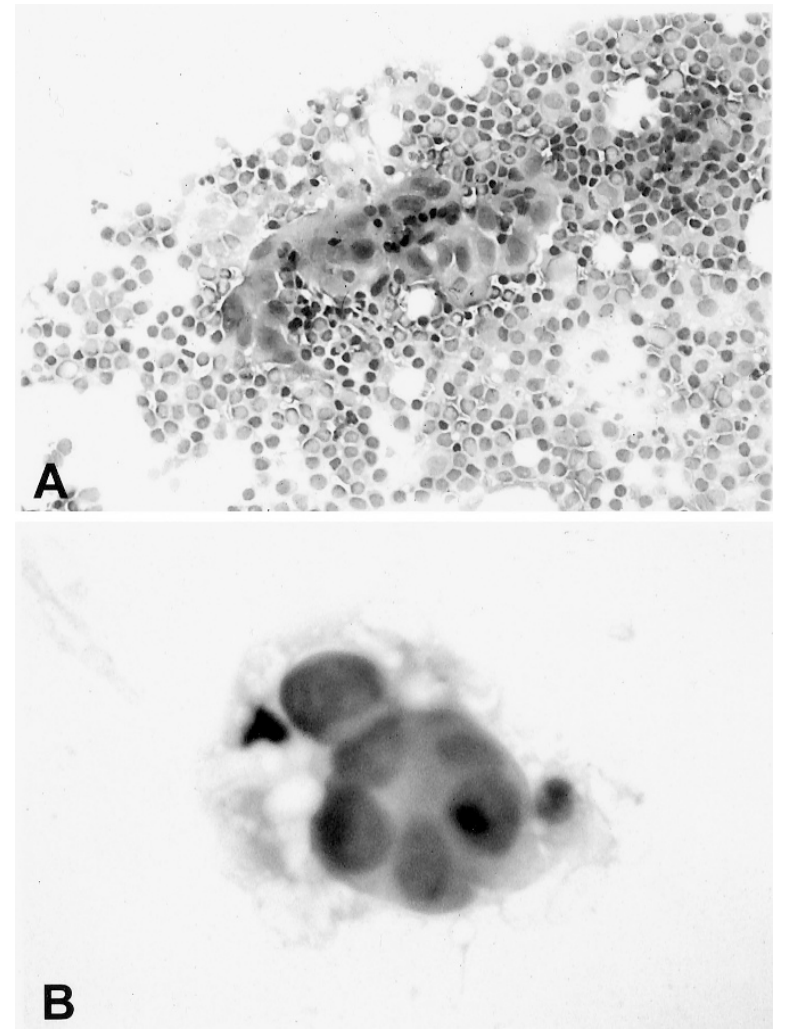

FIGURE 1. Photomicrographs of two false positive sentinel node imprints. A, a cohesive group of cells with increased nuclear to cytoplasmic ratios is present in a background of smaller lymphocytes. This group of cells likely represents a benign aggregate of histiocytes. B, an acinar structure composed of cells with increased nuclear to cytoplasmic ratios is present. These cells represented the only atypical cells in the entire sentinel node and were the basis for the positive diagnosis.

\section{DISCUSSION}

Accurate intraoperative evaluation of sentinel nodes permits an axillary dissection to be performed during the initial operation if the node is positive, saving the patient both the cost and bur- 
den of a second operation. There is no consensus on the optimal for intraoperative evaluation of sentinel lymph nodes $(21,47)$. In 1999, the College of American Pathologists recommended that sentinel node be examined intraoperatively by cytologic methods (48).

Previous sentinel node studies evaluating the use of frozen sectioning have reported variable results with accuracy ranging from $83-98 \%$, sensitivities ranging from $58-87 \%$ and specificity ranging from $99-100 \%$ (47). Several groups have studied the use of intraoperative imprint cytology in the evaluation of sentinel nodes. The accuracy of these studies varies from $78-98 \%$, sensitivity ranges from $29-94 \%$ and specificity ranges from $88-100 \%$ (47). Finally, a few groups have examined the utility of intraoperative evaluation of sentinel nodes using combined frozen sectioning and imprint cytology. The accuracy, sensitivity and specificity of these studies are similar to frozen sectioning or imprint cytology alone $(12,41,42)$. Regardless of the methodology used, the wide disparity between the accuracy of these studies is, in part, due to the non-uniformity of sentinel node examination and, in some studies, the low number of cases examined (47). Overall, sensitivity and accuracy are similar between frozen sectioning and imprint cytologic evaluation. Unfortunately, only a small number of studies directly comparing the accuracy of intraoperative frozen sectioning and imprint cytology exist $(12,41,42)$. Based on the results of these small studies it is not clear if one method is more accurate than the other. In the present study, due to practical limitations of intraoperative evaluation at both an academic hospital and a community hospital, only the use of imprint cytology was performed and a direct comparison between these methodologies could not be made.

False positive intraoperative evaluations of sentinel nodes have been reported using both methodologies, and while these occurrences are rare, they are problematic $(23,29,37,42)$. To minimize the possibility of false-positive results, an indeterminate intraoperative diagnostic category (atypical cells) has been adopted at our laboratory (KRG, EAL, PS, NDP), and is regarded as a negative finding by the surgical staff at the time of surgery. In the early part of the present study, rare cases were diagnosed intraoperatively as (atypical cells). This diagnosis was interpreted by the surgeon as positive resulting in immediate axillary dissection, when in some cases the permanent section histology was negative. Therefore, the indeterminant category was instituted to reduce the false positive rate, albeit at the expense of sensitivity. We feel that any intraoperative evaluation should stress specificity over sensitivity to avoid potentially unnecessary axillary dissection.
Several problem areas exist in intraoperative evaluation of sentinel nodes whether it be by frozen sectioning or by imprint cytology. The first area is the poor sensitivity of detecting micrometastatic disease. In the present study the disparity between detection of micrometastatic disease versus macrometastatic disease was large (Table 3). This disparity also exists in most intraoperative frozen section studies. Although the clinical implications of micrometastatic disease are unclear at present, some have suggested that the use of intraoperative cytokeratin immunohistochemistry protocols may decrease the intraoperative false negative rate. Such intraoperative immunohistochemistry protocols are available using either frozen sections and cytologic imprints $(31,39,40,49)$. At present, frozen section immunohistochemical protocols are employed by at least one group and include complete intraoperative step sectioning of the sentinel node, a process with a reported turnaround time of 65 minutes (31). Alternatively, we are aware of two small studies employing rapid immunohistochemical cytokeratin staining to cytologic imprints of sentinel nodes $(39,40)$. Turnaround time for the immunohistochemical staining in one report was 16 minutes (40). At present it remains to be seen whether immunohistochemical protocols are reliable and fast enough for general use. Any delay in completion of the operative procedure will have a significant impact of the cost of intraoperative analysis. In the present study turnaround time was approximately 20 minutes, and we are not prepared to extend this time for a more rigorous examination. Unfortunately, it is unlikely that the sensitivity of intraoperative evaluation will increase without the use of more rigorous techniques to detect micrometastatic disease. Alternatively, intraoperative evaluation by either method is a reliable detector of macrometastatic disease, which is in and of itself a strong predictor of non-sentinel node metastases (42). In the present study, non-sentinel nodes were significantly more likely to contain metastases if the sentinel node was positive by imprint cytology (38/99 (38\%) versus 13/155 (8\%), $P<.0001$.

If intraoperative evaluation is to be performed, what are acceptable sensitivity and specificity levels? In cohorts of screened patients the sentinel node will be positive in approximately $40 \%$ of patients on final pathology. Even if the sensitivity of intraoperative evaluation is only around $50 \%$, this could potentially save a considerable amount of resources and the burden of extra surgery in a significant number of patients. A cost-benefit analysis was not possible in this retrospective analysis and its determination will be critical for the future direction of intraoperative evaluation of sentinel nodes. The billing charge for imprint cytology at our institution for 2 SLN is \$131 versus \$356 for 
frozen sectioning. This represents a difference of over $\$ 80,000$ in pathology charges alone for the 646 cases in this study.

The second major problem area in intraoperative evaluation of sentinel nodes is the detection of lobular carcinoma. Because of the relative rarity of lobular carcinoma compared with ductal carcinoma, few studies examining intraoperative evaluation of lobular carcinoma, by either frozen section or imprint cytology, are available. The sensitivity of detecting metastatic lobular carcinoma has been as low as $18 \%(35,39,41,50)$. The intraoperative detection of lobular carcinoma is difficult because of its low-grade cytomorphology and its tendency to infiltrate metastatic sites in a single cell or sinus pattern. To our surprise, in the present study no significant difference was observed between the sensitivity of detecting metastatic lobular carcinoma versus ductal carcinoma. Furthermore the sensitivity of detecting micrometastatic disease versus marcometastatic disease was similar to that observed in ductal carcinoma.

In summary, we believe that intraoperative imprint cytology is advantageous over frozen sectioning for many reasons. Imprint cytology is at least as sensitive as frozen sectioning and is as specific. Imprint cytology is less expensive and faster than frozen sectioning. Tissue is not wasted in the cryostat with imprint cytology and the pathologist does not have to attempt to cut lymph nodes with the cryostat that have been largely replaced by fat. Finally, freezing artifact is not introduced into the tissue when imprints are made. Detection of micrometastatic disease is a significant area for improvement and a cost-benefit analysis is needed to determine the direction of future studies. However, we believe that imprint cytology is the preferred method of intraoperative analysis for sentinel nodes found in breast cancer patients.

Acknowledgment: This study was supported, in part, by a grant from the Wake Forest University Comprehensive Cancer Center.

\section{REFERENCES}

1. Albertini JJ, Lyman GH, Cox CE, Yeatman T, Balducci L, Ku $\mathrm{N}$, et al. Lymphatic mapping and sentinel node biopsy in the patient with breast cancer. JAMA 1996;276:1818-22.

2. Giuliano AE, Dale PS, Turner RR, Morton DL, Evans SW, Krasne DL. Improved axillary staging of breast cancer with sentinel lymphadenectomy. Annu Surg 1995;222:394-9.

3. Copeland EM III. Is axillary dissection necessary for $T_{1}$ carcinoma of the breast? J Am Coll Surg 1997;184:397-8.

4. Ruffin WK, Stacey-Clear A, Younger J, Hoover HC. Rationale for routine axillary dissection in carcinoma of the breast. J Am Coll Surg 1995;180:245-51.

5. Graversen HP, Blichert-Toft M, Andersen JA, Zedeler K. Breast cancer: risk of axillary recurrence in node negative patients following partial dissection of the axilla. Eur J Surg Oncol 1988;14:407-12.

6. Fisher B, Redmond C, Fisher ER, Bauer M, Wolmark N, Wickerham DL, et al. Ten-year results of a randomized clinical trial comparing radical mastectomy and total mastectomy with or without radiation. N Engl J Med 1985;312:67481.

7. Renolleau C, Merviel P, Clough KB, Asselain B, Campana F, Durand JC. Isolated axillary recurrences after conservative treatment of breast cancer. Eur J Cancer 1996;32A:617-21.

8. Halverson KJ, Taylor ME, Perez CA, Garcia DM, Myerson R, Philpott G, et al. Regional nodal management and patterns of failure following conservative surgery and radiation therapy for stage I and II breast cancer. Int J Radiat Oncol Biol Phys 1993;26:593-9.

9. Kjaergaard J, Blichert-Toft M, Andersen JA, Pedersen BV. Probability of false negative nodal status in conjunction with partial axillary dissection in breast cancer. Br J Surg 1985;72: 365-7.

10. Hayward JL. The Guy's trial of treatment of early breast cancer. World J Surg 1977;1:314-6.

11. Cabanes PA, Salmon RJ, Vilcoq JR, Durand JC, Fourquet A, Gautier C, et al. Value of axillary dissection in addition to lumpectomy and radiotherapy in early breast cancer. The Breast Carcinoma Collaborative Group of the Institut Curie. Lancet 1992;339:1245-8.

12. van Diest PJ, Torrenga H, Borgstein PJ, Pijpers R, Bleichrodt $\mathrm{RP}$, Rahusen FD, et al. Reliability of intraoperative frozen section and imprint cytological investigation of sentinel lymph nodes in breast cancer. Histopathol 1999;35:14-8.

13. Veronesi U, Paganelli G, Galimberti V, Viale G, Zurrida S, Bedoni M, et al. Sentinel-node biopsy to avoid axillary dissection in breast cancer with clinically negative lymphnodes. Lancet 1997;349:1864-7.

14. Turner RR, Ollila DW, Krasne DL, Giuliano AE. Histopathologic validation of the sentinel node hypothesis for breast carcinoma. Annu Surg 1997;226:271-6.

15. Giuliano AE. Sentinel lymphadenectomy in primary breast carcinoma: an alternative to routine axillary dissection. J Surg Oncol 1996;62:75-6.

16. Koller M, Barsuk D, Zippel D, Engelberg S, Ben-Ari G, Papa MZ. Sentinel lymph node involvement: a predictor for axillary node status with breast cancer: has the time come? Eur J Surg Oncol 1998;24:166-8.

17. Ivens D, Hoe AL, Podd TJ, Hamilton CR, Taylor I, Royle GT. Assessment of morbidity from complete axillary dissection. Br J Cancer 1992;66:136-8.

18. Recht A, Houlihan MJ. Axillary lymph nodes and breast cancer: a review. Cancer 1995;76:1491-512.

19. Morrow M. Role of axillary dissection in breast cancer management. Annu Surg Oncol 1996;3:233-4.

20. Kissin MW, Querci della Rovere G, Easton D, Westbury G. Risk of lymphoedema following treatment of breast cancer. Br J Surg 1986;73:580-4.

21. Schwartz GF, Giuliano AE, Veronesi U. Proceedings of the consensus conference on the role of sentinel lymph node biopsy in carcinoma of the breast, April 19-22, 2001, Philadelphia, Pennsylvania. Cancer 2002;94:2542-51.

22. Rubio IT, Korourian S, Cowan C, Krag DN, Colvert M, Klimberg VS. Use of touch preps for intraoperative diagnosis of sentinel lymph node metastases in breast cancer. Annu Surg Oncol 1998;5:689-94.

23. Ku NN, Ahmad N, Smith PV, Cox CE, Shons A, Reintgen DS, et al. Intraoperative imprint cytology of sentinel lymph nodes in breast cancer [abstract]. Acta Cytol 1997;41:1606-7.

24. Litz C, Miller R, Ewing G, Odell D, Clifford E, Beitsch P. Intraoperative sentinel lymph node touch imprints are not sensitive in detecting metastatic carcinoma [abstract]. Mod Pathol 2000;13:26. 
25. Cserni G. The potential value of intraoperative imprint cytology of axillary sentinel lymph nodes in breast cancer patients. Am Surg 2001;67:86-91.

26. Moes GS, Guibord RS, Weaver DL, Krag DN, Harlow SP. Intraoperative cytologic evaluation of sentinel lymph nodes in breast cancer patients [abstract]. Mod Pathol 2000;13:28.

27. Kane JM III, Edge SB, Winston JS, Watroba N, Hurd TC. Intraoperative pathologic evaluation of a breast cancer sentinel lymph node biopsy as a determinant for synchronous axillary lymph node dissection. Annu Surg Oncol 2001;8:361-7.

28. Ratanawichitrasin A, Biscotti CV, Levy L, Crowe JP. Touch imprint cytological analysis of sentinel lymph nodes for detecting axillary metastases in patients with breast cancer. Br J Surg 1999;86:1346-9.

29. Teng S, Dupont E, McCann C, Wang J, Bolano M, Durand K, et al. Do cytokeratin-positive-only sentinel lymph nodes warrant complete axillary lymph node dissection in patients with invasive breast cancer. Am Surg 2000;66:574-8.

30. Kelley SW, Komorowski RA, Dayer AM. Axillary sentinel lymph node examination in breast carcinoma. Arch Pathol Lab Med 1999;123:533-5.

31. Viale G, Bosari S, Mazzarol G, Galimberti V, Luini A, Veronesi $\mathrm{P}$, et al. Intraoperative examination of axillary sentinel nodes in breast carcinoma patients. Cancer 1999;85:2433-8.

32. Rahusen FD, Pijpers R, van Diest PJ, Bleichrodt RP, Torrenga $\mathrm{H}$, Meijer S. The implementation of the sentinel node biopsy as a routine procedure for patients with breast cancer. Surgery 2000;128:6-12.

33. Weiser MR, Montgomery LL, Susnik B, Tan LK, Borgen PI, Cody HS. Is routine intraoperative frozen-section examination of sentinel lymph nodes in breast cancer worthwhile? Annu Surg Oncol 2000;7:651-5.

34. Flett MM, Going JJ, Stanton PD, Cooke TG. Sentinel node localization in patients with breast cancer. Br J Surg 1998; 85:991-3.

35. Liu LH, Siziopikou KP, Gabram S, McClatchey KD. Evaluation of axillary sentinel lymph node biopsy by immunohistochemistry and multilevel sectioning in patients with breast carcinoma. Arch Pathol Lab Med 2000;124:1670-3.

36. Hill ADK, Tran KN, Akhurst T, Yeung H, Yeh SDJ, Rosen PP, et al. Lessons learned from 500 cases of lymphatic mapping for breast cancer. Annu Surg 1999;229:528-35.

37. Tanis PJ, Boom RPA, Koops HS, Faneyte IF, Peterse JL, Niewig OE, et al. Frozen section investigation of the sentinel node in malignant melanoma and breast cancer. Annu Surg Oncol 2001;8:222-6.

38. Zurrida S, Galimberti V, Orvieto E, Robertson C, Ballardini B, Cremonesi $\mathrm{M}$, et al. Radioguided sentinel node biopsy to avoid axillary dissection in breast cancer. Annu Surg Oncol 2000;7:28-31.

39. Ahmad N, Ku NN, Nicosia SV, Smith PV, Muro-Cacho CA, Livingston SV, et al. Evaluation of sentinel lymph node imprints in breast cancer: role of intraoperative cytokeratin immunostaining in breast cancer staging [abstract]. Acta Cytol 1998;42:1218.

40. Krishnamurthy S, Tarco E, Hunt K, Kuerer H, Ross M, Ames F, et al. Utility of imprint cytology and rapid cytokeratin immunostaining for the intraoperative evaluation of axillary sentinel lymph nodes in breast cancer [abstract]. Mod Pathol 2001;14:29A.

41. Turner RR, Hansen NM, Stern SL, Giuliano AE. Intraoperative examination of the sentinel lymph node for breast carcinoma staging. Am J Clin Pathol 1999;112:627-34.

42. Motomura K, Inaji H, Komoike $\mathrm{Y}$, Kasugai T, Nagumo S, Noguchi S, et al. Intraoperative sentinel node examination by imprint cytology and frozen sectioning during breast surgery. Br J Surg 2000;87:597-601.

43. Flemming ID, Cooper JS, Hensen DE, Hutter RVP, Kennedy BJ, Murphy GP, et al. Breast. In: American Joint Committee on Cancer, editors. Cancer staging manual. 5th edition. Philadelphia: Lippincott-Raven; 1997. pp. 171-80.

44. Elston CW. Grading of invasive carcinoma of the breast. In: Page DL, Anderson TJ, editors. Diagnostic histopathology of the breast. Edinburgh, Scotland: Churchill Livingstone; 1987. pp. 300-11.

45. Hsu SM, Raine L, Fanger H. Use of avidin-biotin-peroxidase complex $(\mathrm{ABC})$ in immunoperoxidase techniques: a comparison between $\mathrm{ABC}$ and unlabelled (PAP) procedures. J Histochem Cytochem 1981;29:577-80.

46. Petris GD, Lev R, Siew S. Peritumoral and nodal muciphages. Am J Surg Pathol 1998;22:545-9.

47. Creager AJ, Geisinger KR. Intraoperative evaluation of sentinel lymph nodes for breast carcinoma: current methodologies. Adv Anat Pathol, 2002;94:3016-22.

48. Fitzgibbons PL, Page DL, Weaver D, Thor AD, Allred DC, Clark GM, et al. Prognostic factors in breast cancer: College of American Pathologists consensus statement. Arch Pathol Lab Med 1999;124:966-78.

49. Chilosi M, Lestani M, Pedron S, Montagna L, Benedetti A, Pizzolo G, et al. A rapid immunohistochemical method for frozen sections. Biotech Histochem 1994;69:235-9

50. Lindsey CA, Grimes MM, Powers CN. Decreased accuracy of sentinel node touch imprints in lobular carcinoma [abstract]. Mod Pathol 2001;14:30A. 